\title{
Isoforms, structures, and functions of versatile spectraplakin MACF1
}

\author{
Lifang $\mathrm{Hu}{ }^{1}$, Peihong Su${ }^{1}$, Runzhi $\mathrm{Li}^{1}$, Chong $\mathrm{Yin}^{1}$, Yan Zhang ${ }^{1}$, Peng Shang ${ }^{1}$, Tuanmin Yang ${ }^{2}$ \& Airong Qian ${ }^{1, *}$ \\ ${ }^{1}$ Key Laboratory for Space Bioscience and Biotechnology, Institute of Special Environmental Biophysics, School of Life Sciences, \\ Northwestern Polytechnical University, Xi'an, Shaanxi 710072, ${ }^{2}$ Honghui Hospital, Xi'an Jiaotong University College of Medicine, Xi'an, \\ Shaanxi 710054, P. R. China
}

\begin{abstract}
Spectraplakins are crucially important communicators, linking cytoskeletal components to each other and cellular junctions. Microtubule actin crosslinking factor 1 (MACF1), also known as actin crosslinking family 7 (ACF7), is a member of the spectraplakin family. It is expressed in numerous tissues and cells as one extensively studied spectraplakin. MACF1 has several isoforms with unique structures and well-known function to be able to crosslink F-actin and microtubules. MACF1 is one versatile spectraplakin with various functions in cell processes, embryo development, tissue-specific functions, and human diseases. The importance of MACF1 has become more apparent in recent years. Here, we summarize the current knowledge on the presence and function of MACF1 and provide perspectives on future research of MACF1 based on our studies and others. [BMB Reports 2016; 49(1): 37-44]
\end{abstract}

\section{INTRODUCTION}

Cytoskeleton in most multicellular organisms is a highly organized and interconnected network of filaments composing of microfilaments (F-actin), microtubules (MTs), and intermediate filaments (IFs). Cytoskeletal network acts as a cytoplasmic scaffold, defining the cell shape, cell mechanical properties, and functions of many other cellular events, including cell polarization, migration, division, adhesion, intracellular trafficking, and organelle locomotion. Thus, cytoskeleton is both static and adaptive, providing a dynamic cellular architecture that can remodel itself to respond to cellular changes. The tight organization and dynamics of these filaments strongly depends on their associated proteins. It has been demonstrated that spectraplakins are cytoskeletal crosslinkers with

${ }^{*}$ Corresponding author. Tel: +86-29-88491840; Fax: +86-2988491840; E-mail: qianair@nwpu.edu.cn

http://dx.doi.org/10.5483/BMBRep.2016.49.1.185

Received 8 September 2015, Revised 12 October 2015, Accepted 27 October 2015

Keywords: Function, Isoform, MACF1, Structure, Spectraplakin ability of interacting with all three types of cytoskeletal filaments, i.e., F-actin, MTs, and IFs (1).

Spectraplakins are exceptionally long and gigantic with molecular weight of $>500 \mathrm{kD}$. They are multi-domain cytoskeletal proteins and master orchestrators for coordinating cytoskeletal elements by binding to F-actin, MTs, and IFs $(1,2)$. Spectraplakins are evolutionarily conserved. They belong to both spectrin and plakin superfamilies (3). "Spectraplakins" are named as combinations of "spectrin" and "plakin" because they share features of both spectrins and plakins $(3,4)$. So far, mammalian spectraplakins have two members: microtubule actin crosslinking factor 1 (MACF1) and bullous pemphigoid antigen 1 (BPAG1). The spectraplakin family also consists of Shot/Kakapo (in Drosophila), Magellan (in zebrafish), and Vab-10 (in Caenorhabditis elegans).

MACF1, also known as actin crosslinking family 7 (ACF7), MACF, macrophin, and trabeculin- $\alpha$, is a widely expressed critical spectraplakin (1). By crosslinking both MTs and F-actin and by controlling the cytoskeletal dynamics, MACF1 plays key roles in the regulation of cell migration (5-8) and cell proliferation and the maintenance of tissue integrity $(9,10)$. In addition, MACF1 mediates signal transduction pivotal for embryo development (11). More recently, a human disease caused by MACF1 mutation has been reported (12).

In this paper, basic molecular characteristics of MACF1 are introduced and current knowledge on MACF1 is reviewed by highlighting its versatile functions. In addition, perspectives on future research of MACF1 are provided based on studies by us and others to provide researchers the current status of MACF1 research.

\section{MACF1: GENE, ISOFORM, AND TISSUE DISTRIBUTION}

\section{Gene and isoforms of MACF1}

MACF1, a large protein with molecular weight of $\sim 600 \mathrm{kD}$, was first discovered by Byers et al. in the effort to screen for additional members of the actin crosslinker superfamily (13). They isolated a partial cDNA of MACF1 using degenerate primer-mediated PCR and named it ACF7. Subsequently, murine ACF7 was further characterized (14) and its full-length cDNA encoding a $608-\mathrm{kD}$ protein was cloned (15). Due to the associ-

ISSN: 1976-670X (electronic edition)

Copyright (c) 2016 by the The Korean Society for Biochemistry and Molecular Biology

(c) This is an open-access article distributed under the terms of the Creative Commons Attribution Non-Commercial License (http://creativecommons.org/licenses/by-nc/4.0) which permits unrestricted non-commercial use, distribution, and reproduction in any medium, provided the original work is properly cited. 
ation of murine ACF7 with both actin and MTs, ACF7 was renamed MACF to represent microtubule actin cross-linking factor. Meanwhile, human cDNA was cloned independently by two groups and named macrophin and trabeculin- $\alpha$, respectively $(16,17)$.

MACF1/ACF7 is encoded by MACF1 gene located on human chromosome 1 p32 or mouse chromosome $4(13,14,18)$. Human MACF1 gene contains at least 102 exons and spans over $270 \mathrm{~kb}$. Genomic organization analysis of human MACF1 gene has demonstrated that MACF1 and ACF7 are the same gene (18). MACF1 gene is a hybrid of genes encoding plakins and spectrins/dystrophins $(15,18)$. MACF1 shares similar gene sequences with plectin, a well-characterized plakin. It has the same exon-intron boundaries for $\mathrm{N}$-terminal actin-binding domain $(\mathrm{ABD})$, one large exon encoding plectin repeats, and a similar serine/glycine-rich C-terminus containing GSR repeats. In addition, the spectrin repeats in MACF1 are similar to those of dystrophin gene. This hybrid gene structure of MACF1 reveals that it is related to Drosophila gene shot and Caenorhabditis elegans gene vab-10. Due to the hybrid structure of these genes and their combined features of both spectrin and plakin family members, they were assigned to the spectraplakin family (3).

Like dystonin/BPAG1 $(19,20)$, MACF1 displays isoform diversity. Several MACF1 isoforms (Fig. 1) produced by alternative splicing and different promoter usage have been reported. Three murine ACF7/MACF1 isoforms with a common $3^{\prime}$ sequence but a unique $5^{\prime}$ region were first identified by Bernier et al. (14). Isoforms ACF7-1 and ACF7-2 contain identical actin-binding domains (ABDs) but different 5'UTRs, while isoform ACF7-3 contains a unique $5^{\prime}$ UTR and a longer $\mathrm{N}$-terminal sequence with just the second half of the ABD (14). Subsequently, a fourth isoform MACF1-4 was cloned (18). Different from the three MACF1 isoforms mentioned above, MACF1-4 has no ABD at the N-terminus. Instead, it has plectin repeats (Fig. 1). Later, another alternatively spliced murine isoform of MACF1 encoding a gigantic protein of approximately 800-kD was revealed (21). It was named MACF1b with the original isoform renamed as MACF1a. MACF1b harbors extra plakin repeats between the plakin domain and the spectrin repeats of MACF1a (Fig. 1). With different N-terminal domains, the first three isoforms ACF7-1, ACF7-2, and ACF7-3 were renamed as MACF1a1, MACF1a2, and MACF1a3, respectively (21). While studying the role of MACF1 in the nervous system by adopting MACF1a conditional knockout (CKO) mice, a novel isoform MACF1c was found by Goryunov et al. (22). MACF1c is identical to MACF1a except that it has no N-terminal ABD domain (Fig. 1).

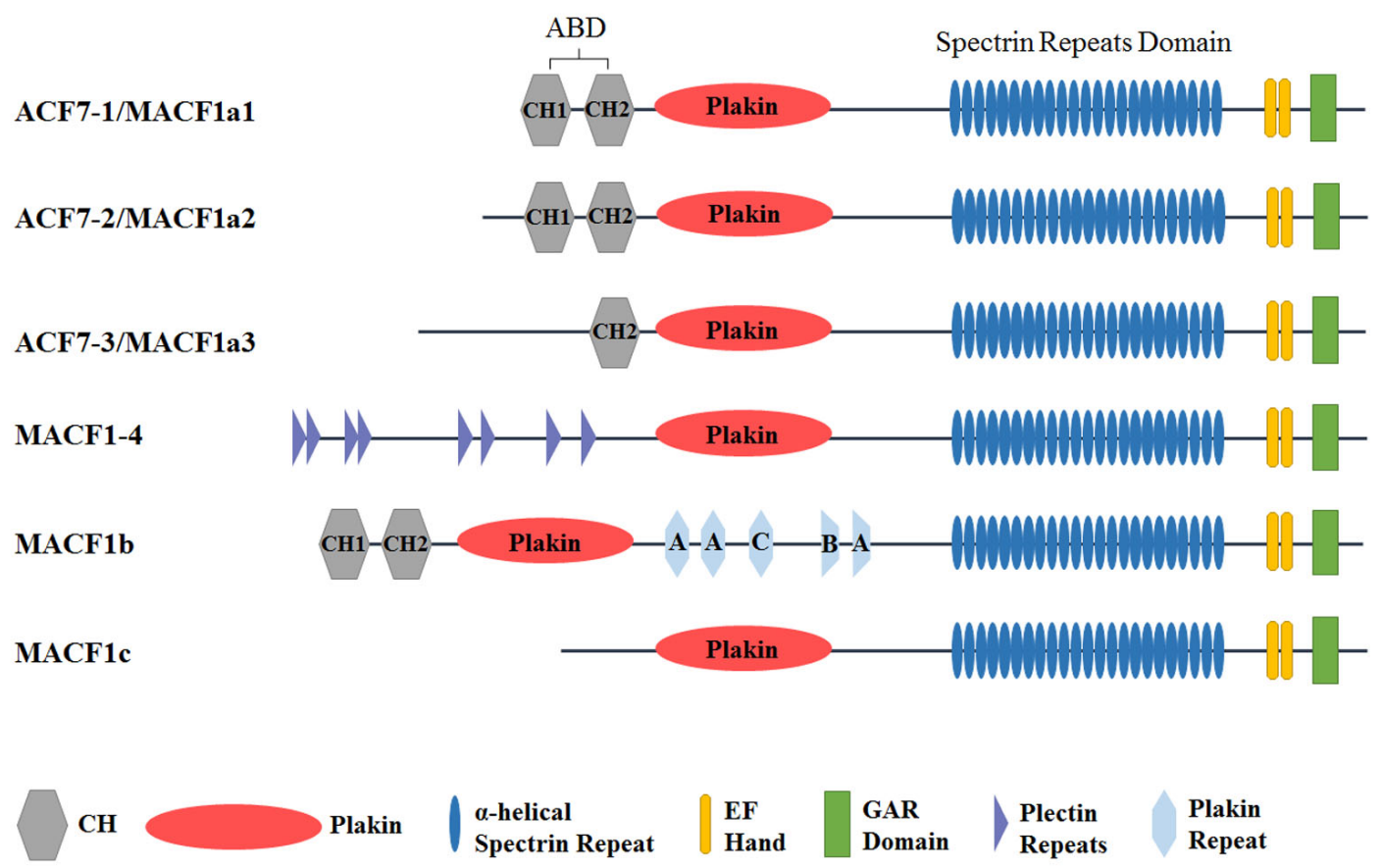

Fig. 1. Isoforms of MACF1 with a combination of different unique domain structure. There are seven main types of functional domains, including an actin binding domain (ABD) with $\mathrm{CH} 1$ and $\mathrm{CH} 2$ fragments, a plakin domain, a spectrin repeats rod domain with $23 \alpha$-helical spectrin repeats, two EF hand motifs, a GAR domain, plectin repeats domain, and plakin repeats domain (PRD). A, B, C represents different types. 
In addition, a putative MACF/MACF1 isoform MACF2 has been reported by Sun et al. (23). Based on sequence homology analysis, a partial human cDNA clone (KIAA0728) encoding a polypeptide sharing $68 \%$ amino acid identities with human MACF was found and called MACF2. However, they found differences in chromosomal locations and nucleotide sequences between MACF and MACF2 transcripts based on unigene database, suggesting that human MACF and MACF2 represent two distinct protein products from two different genes (23). Consequently, MACF2 was demonstrated to be an isoform of BPAG1 and renamed as BPAG1-a (20). Therefore, up to date, a total of six isoforms of MACF1 (MACF1a1, MACF1a2, MACF1a3, MACF1-4, MACF1b, and MACF1c, Fig. 1) have been reported.

\section{MACF1's tissue distribution}

As a cytoskeletal linker protein, MACF1 is ubiquitously expressed in different tissues with some isoforms having different distributions (Table 1).

Bernier et al. were the first to show that MACF1 was widely expressed in postnatal mouse tissues, including brain, spinal cord, spleen, liver, heart, skeletal muscle, stomach, lung, kidney, and skin, with the strongest expression in the lung, followed by brain, spinal cord, cardiac/skeletal muscle, and skin (14). They further demonstrated a predominant expression of MACF1 in neural, muscle, and lung tissue at the beginning of embryonic development which was continued into adulthoods (24). A striking difference in tissue distribution for different MACF1 transcripts has been observed. Relatively higher levels of MACF1a2 have been detected in the brain, spinal cord, and lung, while relatively lower levels of MACF1a2 have been found in the kidney, heart, and skeletal muscles without any detection in the skin, liver, stomach, or the spleen. In contrast, MACF1a1 is predominantly found in the skin, kidney, and stomach. Similarly, MACF1a1 mRNA is detected in embryos from day 7.5 to day 10.5, whereas MACF1a2 mRNA becomes detectable only at day 10.5 . MACF1a3 is predominantly detected in the brain and spinal cord. Moderate levels of MACF1a3 has been found in the skin, lung, and kidney with- out expression in the heart, skeletal muscle, or the liver (24). MACF1b is expressed in all tissues and throughout the development of mouse embryo (21).

MACF1 is widely expressed in human tissues, including pituitary, adrenal, thyroid, salivary gland, mammary glands, pancreas, heart, and skeletal muscle at different levels $(13,16,17)$. MACF1a2 is highly expressed in the brain, heart, lung placenta, liver, kidney, and pancreas (16), while the hybridization signals of MACF1-4 are visible in all tissues, with the strongest signals in the heart, lung, pituitary gland, and placenta (18).

\section{UNIQUE DOMAIN STRUCTURE OF MACF1}

Generally, the structure of MACF1 contains three main domains: an $\mathrm{N}$-terminal domain containing an $\mathrm{ABD}$ and a plakin, a rod domain composed of spectrin repeats, and a C-terminal domain consisting of EF-hand calcium-binding domain and a GAS2-related protein (GAR) domain (23). Seven types of functional domains presenting different combination in different isoforms (Fig. 1) have been found in MACF1.

\section{Actin-binding domain}

MACF1 has an ABD located at the $\mathrm{N}$-terminus, a conserved structure among spectraplakins inherited from members of the spectrin superfamily $(15,25)$. ABD consists of two calponin homology $(\mathrm{CH})$ domains $\mathrm{CH} 1$ and $\mathrm{CH} 2$ that can bind F-actin and enable spectraplakins to interact directly with the actin cytoskeleton. $\mathrm{CH} 1$ alone can bind to actin. $\mathrm{CH} 2$ exerts a weaker binding affinity for actin. However, $\mathrm{CH} 1-\mathrm{CH} 2$ tandem domain has high binding affinity $(26,27)$. Not all MACF1 isoforms have $\mathrm{CH} 1$ and $\mathrm{CH} 2$ domains. MACF1a3 only has $\mathrm{CH} 2$ domain while MACF1-4 and MACF1c have no $\mathrm{CH}$ domain (Fig. 1). Regardless of the $\mathrm{CH}$ domain, the interaction of MACF1 with F-actin is directly affected $(15,25)$. Although the crystal structure of MACF1 has not been reported yet, structure analysis of $\mathrm{ABD}$ in dystrophin and utrophin spectrins has demonstrated an interdomain linker between the $\mathrm{CH} 1$ and $\mathrm{CH} 2$ domains, with the interdomain linker primarily determining

Table 1. The isoforms of MACF1

\begin{tabular}{|c|c|c|c|}
\hline Isoform & Tissue distribution & Domains & References \\
\hline ACF7-1/MACF1a1 & $\begin{array}{l}\text { Broadly expressed with predominance in } \\
\text { skin, kidney, and stomach }\end{array}$ & $\begin{array}{l}\mathrm{ABD}(\mathrm{CH} 1, \mathrm{CH} 2) \text {, Plakin, Spectrin repeats, EF hand, } \\
\text { and GAR. }\end{array}$ & $(14,21,24)$ \\
\hline ACF7-2/MACF1a2 & $\begin{array}{l}\text { Broadly expressed with high level in brain, } \\
\text { spinal cord, and lung }\end{array}$ & $\begin{array}{l}\text { ABD }(\mathrm{CH} 1, \mathrm{CH} 2) \text {, Plakin, Spectrin repeats, EF hand, } \\
\text { and GAR. Different 5' UTR with MACF1a1. }\end{array}$ & $(14,16,21,24)$ \\
\hline ACF7-3/MACF1a3 & Predominant in brain and spinal cord & $\begin{array}{l}\text { Half ABD (CH2), Plakin, Spectrin repeats, EF hand, } \\
\text { and GAR. }\end{array}$ & $(14,21)$ \\
\hline MACF1-4 & $\begin{array}{l}\text { Broadly expressed with high level in heart, } \\
\text { lung, pituitary gland and placenta }\end{array}$ & $\begin{array}{l}\text { Plectin repeats, Plakin, Spectrin repeats, EF hand, } \\
\text { and GAR. }\end{array}$ & $(18)$ \\
\hline MACF1b & Broadly expressed & $\begin{array}{l}\mathrm{ABD}(\mathrm{CH} 1, \mathrm{CH} 2), \text { Plakin, Plakin repeats, Spectrin } \\
\text { repeats, EF hand, and GAR. }\end{array}$ & $(21)$ \\
\hline MACF1c & Nervous system & Plakin, Spectrin repeats, EF hand, and GAR. & $(22)$ \\
\hline
\end{tabular}


the structural stability of tandem $\mathrm{CH}$ domains (28).

\section{Plakin domain}

Following the ABD at the N-terminal of MACF1, there is a plakin domain (23). The plakin domain is also a feature of members of the plakin superfamily (3). It is characterized by a high $\alpha$-helical content (29). Structure analysis of BPAG1 and plectin has revealed a number of spectrin repeats in the plakin domain $(30,31)$, suggesting that the plakin domain is derived from spectrin repeats. The plakin domain is responsible for binding to various adhesion and signaling molecules (e.g. $\beta 4$ integrin, BPAG2, and Erbin) (32-35). It plays a role in the mediation of the binding to cell junctions (29). However, the structure and the function of the plakin domain in MACF1 require further investigation.

\section{Spectrin repeats}

MACF1 contains both the spectrin repeats and the plakin domain. It is classified as a spectraplakin. Spectrin repeats are the typical architecture of the spectrin family. MACF1 has 23 dystrophin-like spectrin repeats (15). Each repeat contains $110 \sim 120$ residues that are folded into three $\alpha$ helices. These helices then form an antiparallel three-helical coiled coil (36, 37). These $\alpha$-helical spectrin repeats then form an extended rod-like structure and act as a spacer region that separates different functional domains at the $\mathrm{N}$ - and $\mathrm{C}$-termini. In addition, these spectrin repeats endow the protein MACF1 with flexibility $(1,3)$.

\section{EF hand}

MACF1 contains two EF-hand calcium-binding motifs located at the C-terminus that are conserved within the spectrin superfamily (23). Sun et al. have indicated that the EF-hand motifs of MACF1 have no effect on the ability of MACF1 to interact with MTs (23).

\section{GAS2-related protein (GAR) domain}

Following the EF-hand motifs, there is a GAR domain located at the C-terminal of MACF1 (Fig. 1). The GAR domain is restricted to spectraplakins. It can be used to distinguish spectraplakins from spectrins and plakins (3). The GAR domain is the homology region shared between Gas2 (growth arrest-specific protein 2) and GAR22 (Gas2 related on chromosome 22) proteins. It has the function to bind and stabilize MTs $(15,23)$.

\section{Plakin repeat domain (PRD)}

PRD is unique to members of the plakin family. It only exists in the MACF1b isoform (21). Based on well-characterized A, $B$, and $C$ type PRDs in desmoplakin $(38,39)$, Lin et al. have revealed five PRDs in MACF1b, including two A type, one C type, one incomplete $B$ type, and one incomplete A type (21). They have further demonstrated that PRD targets MACF1b to the Golgi complex via its N-terminal portion of two consecutive A-type subdomains.

\section{Plectin repeats}

Plectin repeats are unique to the MACF1-4 isoform (18). MACF1-4 contains eight plectin repeats instead of $A B D$ at the $\mathrm{N}$-terminus (Fig. 1). Studies from the plakins that contain plectin repeats have demonstrated the essential role of plectin repeats in binding IFs. Therefore, the same function of plectin repeats may exist in MACF1-4. This requires further study.

\section{MACF1 IN CELL MIGRATION}

MACF1 has a well-known function in cell migration by coordinating F-actin and MTs dynamics (5-7). MACF1 binds to both F-actin and MTs with its ABD and MT-binding domains. In endodermal cells, MACF1 can bind along MTs, especially at the plus-ends of those MTs toward the edges of migrating cells (5, 25). Loss of MACF1 causes perturbations in MTs trajectories, dynamic stabilities, and tracking ability along actin cables, which can affect cell migration in response to wounding (5). MACF1 deficiency compromises the targeting of MTs along F-actin to focal adhesions (FAs), stabilizes FA-actin networks, and impairs epidermal migration (6). The underlying mechanisms involve the F-actin binding domains in MACF1 and an intrinsic actin-regulated ATPase domain of MACF1 for the regulation of cell migration. Therefore, there is a new functional domain in MACF1. MACF1 also sustains directional cell migration in stem cells responsible for homeostasis and wound healing (7). It has been demonstrated that GSK3 $\beta$ can phosphorylate and uncouple MACF1 from MTs during the regulation of cell migration by MACF1 (7). Moreover, MACF1 can regulate cell migration in neurons (8) with several partners, including ErbB2 receptor and ELMO $(40,41)$.

\section{MACF1 IN CELL SIGNALING}

Given the implication that MACF $1^{-/-}$mice and $\mathrm{Wnt} 3^{-/-}$and Lrp5/6 double-knockout mice have similar phenotype, the role of MACF1 in Wnt/ $\beta$-catenin signal transduction has been determined by Chen et al. (11). Their results have indicated that MACF1 can interact with the Axin complex, including Axin, GSK-3 $\beta, \beta$-catenin, and APC. They have also revealed that MACF1 is responsible for the translocation of the Axin complex from the cytoplasm to the cell membrane where MACF1 also binds to co-receptor LRP5/6. Reduction of MACF1 will impair the translocation of the Axin complex, thus inhibiting Wnt-induced TCF/ $\beta$-catenin-dependent transcriptional activation. In addition, MACF1 has a phosphorylation site for GSK-3 $\beta$ (7). It can also mediate GSK-3 signaling (8). These findings have demonstrated a role of MACF1 in cell signaling. In addition, MACF1 functions in vesicles transport. In axonal vesicle transportation, MACF1 acts as a medium to transport vesicles from the trans-Golgi network to Kif5A. Malfunction of MACF1 can cause vesicles incapable of being mobilized to the cell periphery (42). Interaction between Trans-Golgi protein p230 and MACF1 can mediate the transport of mAtg9 from the trans- 
Golgi network to peripheral phagophores in the early step of autophagy (43).

\section{MACF1 IN EMBRYO}

As a ubiquitously-expressed protein, MACF1 is present at embryonic day 7.5 (E7.5) in mouse (24), which is quite early, suggesting that MACF1 may act as a critical molecule during embryo development. Chen et al. have confirmed the essential role of MACF1 in embryo development using MACF1 $1^{-1-}$ mice (11). The MACF1 $1^{-1-}$ mice died at the gastrulation stage with a "head-without-trunk". They had developmental retardation at E7.5, lacking primitive streak, node, and mesoderm, similar to the phenotypes of $W n t 3^{-1-}$ embryos (44) and LRP5/6 double-knockout embryos (45). Moreover, a deletion mutation in the magellan gene encoding MACF1 in zebrafish caused oocyte polarization failure (46). Taken together, these data demonstrate the importance of MACF1 in embryo development.

\section{MACF1 IN THE NERVE}

High levels of MACF1 and significant physiological function of the homolog of MACF1 Kakapo/Shot in the nervous system $(15,47)$ indicate that MACF1 might play important role in the nervous system. By generating a nervous system-specific Macf1 knockout (cKO) mouse model, Goryunov et al. (22) have underscored the importance of MACF1 in nervous system development. MACF1 cKO brains have been found to have multiple neuronal developmental defects (22). Defects in neuronal migration in the $\mathrm{CKO}$ cortex are also observed, suggesting a critical role of MACF1 in neuronal migration (22). In addition, the MACF1c isoform that lacks an ABD compared to MACF1a cannot compensate for the loss of MACF1a (22). Their findings indicate the necessity to interact with actin for MACF1's function in the nervous system. Other studies have confirmed the role of MACF1 in the regulation of neuronal growth, survival, and migration $(8,48,49)$. These evidences indicate the importance of MACF1 in the nerve.

\section{MACF1 IN SKIN}

The physiological function of ACF7/MACF1 in mammalian skin has been uncovered mainly by Elaine Fuchs' group. Karakesisoglou et al. first showed the expression of MACF1 in the epidermis of mice and identified the functional binding sites of MACF1 for both actin and MTs (25). Subsequently, Wu et al. elucidated the function of MACF1 in the skin by conditionally knocking out (CKO) MACF1 in epidermal cells $(6,7)$. No gross morphological change in the skin or hair coat was observed in MACF1 CKO mice. However, a significant delay in repairing full-thickness wounds due to defects in epidermal cell migration was detected in the skin of CKO mice in response to injury. Further studies revealed that cell migration defects were rooted in perturbations in epidermal-ECM adhe- sion caused by a malfunction in focal adhesion dynamics due to MACF1 absence (6). Moreover, a link between MACF1 and GSK3 $\beta$ controlling skin stem cell migration and polarized locomotion upon injury was identified (7). Taken together, these results suggest a key role of MACF1 in maintaining skin integrity and regulating epidermal cell migration through coordinating with F-actin and MTs dynamics. Recently, MACF1 has been linked to cellular processes involved in human epidermal cells in response to extremely-low-frequency electric fields (50).

\section{MACF1 IN BONE}

MACF1 is a widely expressed protein. However, its role in bone tissue is not well understood. Our group have focused on the role of MACF1 in bone and reported some primary findings in osteoblasts. MACF1 is widely expressed in osteoblastic cells. It partially co-localizes with F-actin and MTs. It is scattered in the cytoplasm and cell periphery. MACF1 also participates in the osteoblast in response to environmental stimuli (51). Under diamagnetic levitation conditions, the distribution of MACF1 in osteoblasts was altered and the co-localization of MACF1 with F-actin and its co-alignment with MTs were also changed (51). Our further studies have demonstrated that MACF1 is crucial for maintaining cell shape and cell proliferation in osteoblasts (52). Knockdown of MACF1 can induce large cells with a binuclear or multinuclear structure, disrupt the organization of F-actin and MTs, and inhibit osteoblast proliferation (52). These data indicate that MACF1 is important in osteoblasts and bone.

\section{MACF1 IN OTHER TISSUES}

MACF1 also plays important roles in the heart, colony, and other tissues $(53,54)$. Fassett et al. (53) have identified a role of MACF1 in regulating cardiomyocyte MTs distribution and adaptation to hemodynamic overload for the first time using inducible cardiac-specific MACF1 knockout (KO) mice. The heart size or function of MACF1 KO mice was not affected under basal conditions. However, exacerbated pressure overload induced left ventricular (LV) hypertrophy, LV dilation, and contractile dysfunction (53). This manifestation has been found to be related to altered MT distribution and several relevant signaling proteins caused by the absence of MACF1 (53). MACF1 is also critical to the maintenance of colonic paracellular permeability because cKO of MACF1 causes significant decrease in colonic mucosal permeability associated with disturbed epithelial arrangement and cytoskeleton dysregulation (54).

\section{MACF1 AND HUMAN DISEASE}

In 2014, a human disease caused by a mutation in MACF1 was reported (12). This disease was found in a 12-year-old boy (the proband) who suffered from a skeletal muscle problems 
with mainly manifestation of generalized hypotonia and pain. Genetic analysis revealed a single duplicated region on chromosome $1 \mathrm{p} 34.3$ covering a large part of the MACF1 gene. This affected all 4 major MACF1 isoforms in the proband. Further studies demonstrated that the duplication resulted in a remarkable reduction in MACF1 expression, resulting in reduced motility and internal structural changes of endothelial and satellite cells in the skeletal muscle in the proband. This novel myopathy caused by the duplication in MACF1 was named spectraplakinopathy type 1 (12). The discovery of this MACF1-related genetic human disease has shed light on the importance of MACF1 in human.

Recently, it was found that MACF1 was involved in cancer. A genetic alteration of MACF1 has been detected in numerous cancers, including breast cancer, colorectal cancer, gliomas, and others cancers (55-59). Although the function of MACF1 in cancer requires further investigation, these data suggest that MACF1 might be used as a marker for cancer diagnosis and cancer therapy.

\section{CONCLUSIONS AND FUTURE PERSPECTIVES}

In this review, we summarized the current knowledge of MACF1. As an extensively studied mammalian spectraplakin, MACF1 is broadly expressed with versatility. It has different isoforms, structures, and functions. Similar to other spectraplakins, MACF1 has several isoforms expressed in different tissues. MACF1 participates in many cellular processes and specific-tissue functions. MACF1 is crucial to cell polarization and migration by regulating the dynamics of F-actin and MTs. In addition, MACF1 plays a key role in mediating cellular signaling transduction. The embryonic lethality due to a lack of MACF1 has demonstrated its essential role in embryo development. In addition, MACF1's ubiquitous expression in all tissue indicates its physiological significance. In fact, MACF1 plays critical roles in controlling the normal development of the nervous system, maintaining skin integrity and colonic paracellular permeability, and adapting cardiomyocyte to hemodynamic overload. Moreover, our findings suggest a novel role of MACF1 in bone tissue. More importantly, mutation in MACF1 has been described in a novel myopathy disease in humans and cancer development. Thus, MACF1 is attracting more attention.

Although MACF1's functions have been extensively studied and uncovered, many aspects of MACF1 have not been well understood. For example, how is the MACF1 expression regulated? What is the role of MACF1 with its ATPase activity? What is the exact role of MACF1 in cancer development? Considering the fact that MACF1 is involved in both osteoblast function and $\mathrm{Wnt} / \beta$-catenin signal transduction critical for the regulation of osteoblast differentiation and bone formation, it will be interesting to see whether MACF1 can control bone formation via $\mathrm{Wnt} / \beta$-catenin signaling. Further study of MACF1 is merited to unravel interesting and exciting new functions of
MACF1 in both physiological and pathological settings.

\section{ACKNOWLEDGEMENTS}

This study is partly funded by the National Natural Science Foundation of China (31400725, 31570940), Project Funded by China Postdoctoral Science Foundation (2014M562450, 2015T81051), Project Supported by Natural Science Basic Research Plan in Shaanxi Province of China (2015JQ3076), and Fundamental Research Funds for the Central Universities (3102014JKY15007).

\section{REFERENCES}

1. Suozzi KC, Wu X and Fuchs E (2012) Spectraplakins: master orchestrators of cytoskeletal dynamics. J Cell Biol 197, 465-475

2. Huelsmann S and Brown NH (2014) Spectraplakins. Curr Biol 24, R307-308

3. Roper K, Gregory SL and Brown NH (2002) The 'spectraplakins': cytoskeletal giants with characteristics of both spectrin and plakin families. J Cell Sci 115, 4215-4225

4. Sonnenberg A and Liem RK (2007) Plakins in development and disease. Exp Cell Res 313, 2189-2203

5. Kodama A, Karakesisoglou I, Wong E, Vaezi A and Fuchs E (2003) ACF7: an essential integrator of microtubule dynamics. Cell 115, 343-354

6. Wu X, Kodama A and Fuchs E (2008) ACF7 regulates cytoskeletal-focal adhesion dynamics and migration and has ATPase activity. Cell 135, 137-148

7. Wu X, Shen QT, Oristian DS et al (2011) Skin stem cells orchestrate directional migration by regulating microtubule-ACF7 connections through GSK3 $\beta$. Cell 144, 341-352

8. Ka M, Jung EM, Mueller U and Kim WY (2014) MACF1 regulates the migration of pyramidal neurons via microtubule dynamics and GSK-3 signaling. Dev Biol 395, 4-18

9. Roper K and Brown NH (2003) Maintaining epithelial integrity: a function for gigantic spectraplakin isoforms in adherens junctions. J Cell Biol 162, 1305-1315

10. Bouameur JE, Favre B and Borradori L (2014) Plakins, a versatile family of cytolinkers: roles in skin integrity and in human diseases. J Invest Dermatol 134, 885-894

11. Chen HJ, Lin CM, Lin CS, Perez-Olle R, Leung $C L$ and Liem RK (2006) The role of microtubule actin cross-linking factor 1 (MACF1) in the Wnt signaling pathway. Genes Dev 20, 1933-1945

12. Jorgensen LH, Mosbech MB, Faergeman NJ, Graakjaer J, Jacobsen SV and Schroder HD (2014) Duplication in the microtubule-actin cross-linking factor 1 gene causes a novel neuromuscular condition. Sci Rep 4, 5180

13. Byers TJ, Beggs AH, McNally EM and Kunkel LM (1995) Novel actin crosslinker superfamily member identified by a two step degenerate PCR procedure. FEBS Lett 368, 500-504

14. Bernier G, Mathieu M, De Repentigny $Y$, Vidal SM and Kothary R (1996) Cloning and characterization of mouse ACF7, a novel member of the dystonin subfamily of actin 
binding proteins. Genomics 38, 19-29

15. Leung $C L$, Sun D, Zheng $M$, Knowles DR and Liem RK (1999) Microtubule actin cross-linking factor (MACF): a hybrid of dystonin and dystrophin that can interact with the actin and microtubule cytoskeletons. J Cell Biol 147, 1275-1286

16. Okuda T, Matsuda S, Nakatsugawa S et al (1999) Molecular cloning of macrophin, a human homologue of Drosophila kakapo with a close structural similarity to plectin and dystrophin. Biochem Biophys Res Commun 264, 568-574

17. Sun Y, Zhang J, Kraeft SK et al (1999) Molecular cloning and characterization of human trabeculin-alpha, a giant protein defining a new family of actin-binding proteins. J Biol Chem 274, 33522-33530

18. Gong TW, Besirli CG and Lomax MI (2001) MACF1 gene structure: a hybrid of plectin and dystrophin. Mamm Genome 12, 852-861

19. Brown A, Bernier G, Mathieu M, Rossant J and Kothary R (1995) The mouse dystonia musculorum gene is a neural isoform of bullous pemphigoid antigen 1 . Nat Genet 10, 301-306

20. Leung $C L$, Zheng M, Prater SM and Liem RK (2001) The BPAG1 locus: Alternative splicing produces multiple isoforms with distinct cytoskeletal linker domains, including predominant isoforms in neurons and muscles. J Cell Biol 154, 691-697

21. Lin $\mathrm{CM}$, Chen $\mathrm{HJ}$, Leung $\mathrm{CL}$, Parry $\mathrm{DA}$ and Liem RK (2005) Microtubule actin crosslinking factor $1 \mathrm{~b}$ : a novel plakin that localizes to the Golgi complex. J Cell Sci 118, 3727-3738

22. Goryunov D, He CZ, Lin CS, Leung CL and Liem RK (2010) Nervous-tissue-specific elimination of microtubule-actin crosslinking factor $1 \mathrm{a}$ results in multiple developmental defects in the mouse brain. Mol Cell Neurosci 44, 1-14

23. Sun D, Leung CL and Liem RK (2001) Characterization of the microtubule binding domain of microtubule actin crosslinking factor (MACF): identification of a novel group of microtubule associated proteins. J Cell Sci 114, 161-172

24. Bernier G, Pool M, Kilcup M, Alfoldi J, De Repentigny $Y$ and Kothary R (2000) Acf7 (MACF) is an actin and microtubule linker protein whose expression predominates in neural, muscle, and lung development. Dev Dyn 219, 216-225

25. Karakesisoglou I, Yang Y and Fuchs E (2000) An epidermal plakin that integrates actin and microtubule networks at cellular junctions. J Cell Biol 149, 195-208

26. Way M, Pope B and Weeds AG (1992) Evidence for functional homology in the F-actin binding domains of gelsolin and alpha-actinin: implications for the requirements of severing and capping. J Cell Biol 119, 835-842

27. Winder SJ, Hemmings L, Maciver SK et al (1995) Utrophin actin binding domain: analysis of actin binding and cellular targeting. J Cell Sci 108 (Pt 1), 63-71

28. Bandi S, Singh SM and Mallela KM (2015) Interdomain linker determines primarily the structural stability of dystrophin and utrophin tandem calponin-homology domains rather than their actin-binding affinity. Biochemistry 54, 5480-5488

29. Jefferson JJ, Leung CL and Liem RK (2004) Plakins: goliaths that link cell junctions and the cytoskeleton. Nat
Rev Mol Cell Biol 5, 542-553

30. Jefferson JJ, Ciatto C, Shapiro L and Liem RK (2007) Structural analysis of the plakin domain of bullous pemphigoid antigen1 (BPAG1) suggests that plakins are members of the spectrin superfamily. J Mol Biol 366, 244-257

31. Sonnenberg A, Rojas AM and de Pereda JM (2007) The structure of a tandem pair of spectrin repeats of plectin reveals a modular organization of the plakin domain. J Mol Biol 368, 1379-1391

32. Rezniczek GA, de Pereda JM, Reipert S and Wiche $G$ (1998) Linking integrin $\alpha 6 \beta 4$-based cell adhesion to the intermediate filament cytoskeleton: direct interaction between the $\beta 4$ subunit and plectin at multiple molecular sites. J Cell Biol 141, 209-225

33. Koster J, Geerts D, Favre B, Borradori L and Sonnenberg A (2003) Analysis of the interactions between BP180, BP230, plectin and the integrin $\alpha 6 \beta 4$ important for hemidesmosome assembly. J Cell Sci 116, 387-399

34. Hopkinson SB and Jones JC (2000) The $\mathrm{N}$ terminus of the transmembrane protein BP180 interacts with the $\mathrm{N}$-terminal domain of BP230, thereby mediating keratin cytoskeleton anchorage to the cell surface at the site of the hemidesmosome. Mol Biol Cell 11, 277-286

35. Favre B, Fontao L, Koster J et al (2001) The hemidesmosomal protein bullous pemphigoid antigen 1 and the integrin $\beta 4$ subunit bind to ERBIN. Molecular cloning of multiple alternative splice variants of ERBIN and analysis of their tissue expression. J Biol Chem 276, 32427-32436

36. Yan Y, Winograd E, Viel A, Cronin T, Harrison SC and Branton D (1993) Crystal structure of the repetitive segments of spectrin. Science 262, 2027-2030

37. Pascual J, Pfuhl $M$, Walther D, Saraste $M$ and Nilges $M$ (1997) Solution structure of the spectrin repeat: a left-handed antiparallel triple-helical coiled-coil. J Mol Biol 273, 740-751

38. Choi HJ, Park-Snyder S, Pascoe LT, Green KJ and Weis WI (2002) Structures of two intermediate filament-binding fragments of desmoplakin reveal a unique repeat motif structure. Nat Struct Biol 9, 612-620

39. Green KJ, Parry DA, Steinert PM et al (1990) Structure of the human desmoplakins. Implications for function in the desmosomal plaque. J Biol Chem 265, 2603-2612

40. Zaoui K, Benseddik K, Daou P, Salaun D and Badache A (2010) ErbB2 receptor controls microtubule capture by recruiting ACF7 to the plasma membrane of migrating cells. Proc Natl Acad Sci U S A 107, 18517-18522

41. Margaron Y, Fradet N and Cote JF (2013) ELMO recruits actin cross-linking family 7 (ACF7) at the cell membrane for microtubule capture and stabilization of cellular protrusions. J Biol Chem 288, 1184-1199

42. Burgo A, Proux-Gillardeaux V, Sotirakis E et al (2012) A molecular network for the transport of the TI-VAMP/VAMP7 vesicles from cell center to periphery. Dev Cell 23, 166-180

43. Sohda M, Misumi Y, Ogata S et al (2015) Trans-Golgi protein p230/golgin-245 is involved in phagophore formation. Biochem Biophys Res Commun 456, 275-281

44. Liu P, Wakamiya M, Shea MJ, Albrecht U, Behringer RR and Bradley A (1999) Requirement for Wnt3 in vertebrate axis formation. Nat Genet 22, 361-365

45. Kelly OG, Pinson KI and Skarnes WC (2004) The Wnt co-receptors Lrp5 and Lrp6 are essential for gastrulation in 
mice. Development 131, 2803-2815

46. Gupta T, Marlow FL, Ferriola D et al (2010) Microtubule actin crosslinking factor 1 regulates the Balbiani body and animal-vegetal polarity of the zebrafish oocyte. PLoS Genet 6, e1001073

47. Prokop A, Uhler J, Roote J and Bate M (1998) The kakapo mutation affects terminal arborization and central dendritic sprouting of Drosophila motorneurons. J Cell Biol 143, 1283-1294

48. Sanchez-Soriano N, Travis M, Dajas-Bailador F, GoncalvesPimentel C, Whitmarsh AJ and Prokop A (2009) Mouse ACF7 and drosophila short stop modulate filopodia formation and microtubule organisation during neuronal growth. J Cell Sci 122, 2534-2542

49. Munemasa Y, Chang CS, Kwong JM et al (2012) The neuronal EGF-related gene Nell2 interacts with Macf1 and supports survival of retinal ganglion cells after optic nerve injury. PLoS One 7, e34810

50. Collard JF and Hinsenkamp M (2015) Cellular processes involved in human epidermal cells exposed to extremely low frequency electric fields. Cell Signal 27, 889-898

51. Qian AR, Hu LF, Gao X et al (2009) Large gradient high magnetic field affects the association of MACF1 with actin and microtubule cytoskeleton. Bioelectromagnetics 30, 545-555

52. Hu LF, Su PH, Li RZ et al (2015) Knockdown of micro- tubule actin crosslinking factor 1 inhibits cell proliferation in MC3T3-E1 osteoblastic cells. BMB Rep 48, 583-588

53. Fassett JT, Xu X, Kwak D et al (2013) Microtubule Actin Cross-linking Factor 1 regulates cardiomyocyte microtubule distribution and adaptation to hemodynamic overload. PLoS One 8, e73887

54. Liang Y, Shi C, Yang J et al (2013) ACF7 regulates colonic permeability. Int J Mol Med 31, 861-866

55. Sjoblom T, Jones S, Wood LD et al (2006) The consensus coding sequences of human breast and colorectal cancers. Science 314, 268-274

56. Zhang J, Wu G, Miller CP et al (2013) Whole-genome sequencing identifies genetic alterations in pediatric lowgrade gliomas. Nat Genet 45, 602-612

57. Fleischer T, Frigessi A, Johnson KC et al (2014) Genomewide DNA methylation profiles in progression to in situ and invasive carcinoma of the breast with impact on gene transcription and prognosis. Genome Biol 15, 435

58. Del Valle PR, Milani C, Brentani MM et al (2014) Transcriptional profile of fibroblasts obtained from the primary site, lymph node and bone marrow of breast cancer patients. Genet Mol Biol 37, 480-489

59. Stransky N, Cerami E, Schalm S, Kim JL and Lengauer C (2014) The landscape of kinase fusions in cancer. Nat Commun 5, 4846 